\title{
Local Atomic Distortions in Strontium Cobaltite Due to Oxygen Vacancies
}

\author{
Matthew F. Chisholm, Hyoung Jeen Jeen, and Ho Nyung Lee
}

Materials Science and Technology Division, Oak Ridge National Laboratory, Oak Ridge, Tennessee 37831, USA

Strontium cobaltite, $\mathrm{SrCoO}_{x}$, appears to be an excellent platform for the study of oxidation effects in ionic materials and resulting property changes. These materials exhibit highly contrasting electronic and magnetic ground states depending on the Co oxidation state, reflecting the crucial role of the oxygen content in determining the physical properties of transition metal oxides.[1,2] Improved understanding of oxygen incorporation in this cobaltite will provide a pathway to the design of new ionic materials, in which the oxidation state can be tuned for desired energy generation, storage, and electrochemical sensing applications.

In this study, epitaxially stabilized $\mathrm{SrCoO}_{2.5}$ (SCO) thin films were grown on (001) $\mathrm{SrTiO}_{3}$ (STO) using pulsed laser epitaxy. Bulk SCO has an orthorhombic crystal structure $\left(\mathrm{a}_{\mathrm{o}}=5.5739, \mathrm{~b}_{\mathrm{o}}=\right.$ 5.4697, and $\left.\mathrm{c}_{\mathrm{o}}=15.7450 \AA\right)$. [3] If represented with a pseudo-tetragonal basis $\left(\mathrm{a}_{\mathrm{t}}=3.905\right.$ and $\mathrm{c}_{\mathrm{t}} / 4=$ $3.9363 \AA)$, the cobaltite film is seen to be lattice matched with the STO substrate ( $\mathrm{a}=3.905 \AA)$.

Figure 1 shows cross-sectional high-angle annular dark-field images of orthogonal views of the SCO film on STO as viewed along the $<110>$ STO directions. These images were obtained using a fifthorder aberration-corrected Nion UltraSTEM 200 operated at $200 \mathrm{kV}$. While the [100] view of the film looks similar to that of the STO substrate, the [010] view clearly reveals the alternating stack of fully oxygenated octahedral and oxygen-deficient tetrahedral sub-layers (see the inset in Fig. 1 for schematics of the brownmillerite crystal structure) that are present in this material. The local structural changes due to the oxygen deficiency are quantified in Figure 2. It is seen that: (1) the CoCo lateral atomic spacing modulates within the tetrahedral layers owing to the volume expansion near oxygen vacancy sites; (2) there is a $\sim 30 \%$ increase in the vertical $\mathrm{Sr}-\mathrm{Sr}$ atomic spacing in the oxygen deficient tetrahedral layers $\left(\mathrm{c}^{\prime} / 2=4.3 \pm 0.1 \AA\right)$ over that in the octahedral layers $\left(\mathrm{c}^{\prime \prime} / 2=3.4 \pm\right.$ $0.1 \AA)$; and (3) there is a local modulation of the lateral $\mathrm{Sr}-\mathrm{Sr}$ atomic spacing $(2.8 \pm 0.1$ and $2.6 \pm$ $0.1 \AA$ ), which is induced by a local tilt of the material's oxygen octahedra. We note that the octahedral tilt in SCO has never been visualized previously at the atomic scale.

The collective displacements of Co ions in the tetrahedral layers create well-ordered 1D vacancy channels. It is expected that such well-ordered vacancy channels will offer an interesting playground for the study of fast ion conductivity because it is known that good ionic conductors contain large open frameworks. Oxygen content control is of interest for both the underlying physics and technological applications of multivalent oxides. For example, rapid, reversible redox activity should be possible by moving oxygen into and out of these materials.[4-5]

References

[1] Y. Long et al. J. Phys.: Condens. Mat. 23, (2011), 245601-245606.

[2] T. Takeda et al. J. Phys. Soc. Jpn. 33 (1972), 970-972.

[3] A. Muñoz, et al. Phys. Rev. B 10 (2011), 278

[4] H. Jeen et al. submitted to Nature Materials Jan. 2013. 
[5] This work was supported by the DOE Office of Basic Energy Sciences, Materials Sciences and Engineering Division.

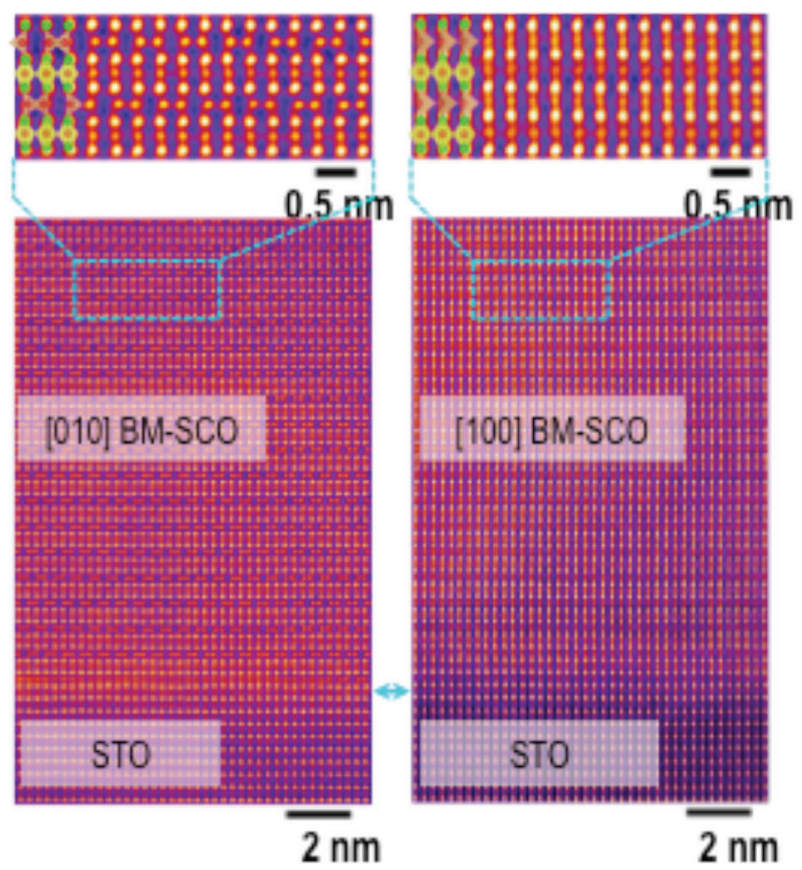

Figure 1. Cross sectional HAADF images of the two domains of SCO on STO as viewed along the $<110>$ direction of STO. The inserts above show magnified views of the SCO film and schematics of the atomic structure.

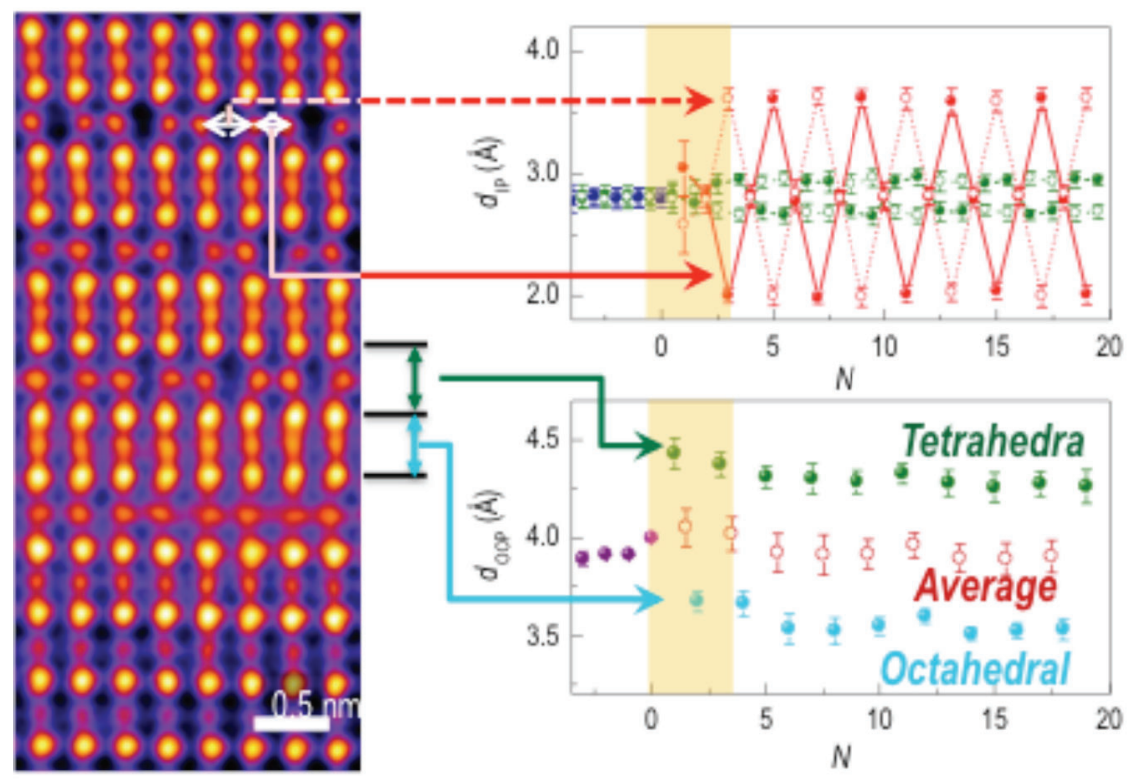

Figure 2. Atomic distortions in SCO grown on STO as measured from the HAADF image. The top panel shows the in-plane (horizontal) distances between the B-site columns (Co and Ti) that reveal cation-channels open with oxygen vacancy ordering. The bottom panel show the vertical distances between Sr columns again showing the expansion of the cation separation with the presence of oxygen vacancies on alternating Co-O planes. 